\title{
De l'engouement à la rupture. Les Juifs
} communistes à Bruxelles et le PCB (1944-1956)

\section{Arnaud Bozzini}

\section{(2) OpenEdition}

12 Journals

Édition électronique

URL : http://journals.openedition.org/cmc/802

DOI : $10.4000 / \mathrm{cmc} .802$

ISSN : 2684-3080

\section{Éditeur}

Fondation de la Mémoire Contemporaine

\section{Édition imprimée}

Date de publication : 1 janvier 2006

Pagination : 131-158

ISSN : 1377-1256

\section{Référence électronique}

Arnaud Bozzini, « De l'engouement à la rupture. Les Juifs communistes à Bruxelles et le PCB

(1944-1956) », Les Cahiers de la Mémoire Contemporaine [En ligne], 7| 2006, mis en ligne le 01 octobre 2020, consulté le 30 octobre 2020. URL : http://journals.openedition.org/cmc/802 ; DOI : https:// doi.org/10.4000/cmc.802 


\title{
De l'engouement à la rupture Les J uifs communistes à B ruxelles et le PCB (1944-1956)
}

\author{
Arnaud Bozzini
}

Au printemps 1946, lorsque Berthe Berger, responsable nationale du "travail juif" au Parti communiste de B elgique (PCB), constate que les rapports entre le P CB et le P oale T si on de gauche (parti sioniste) ne cessent de se dégrader, elle en fait part à la direction du Parti ${ }^{1}$. Elle soumet l'idée d'envoyer chez les sionistes de gauche « un sous-marin »2. Son choix se porte sur Chlioma Fridman, militant de longue date d'origine bessarabienne3. Mis au courant de l'idée de sa camarade, le dirigeant du PCB Hertz J ospa émet certains doutes. Selon lui, Mikado, nom de guerre de Chlioma F ridman, n'est pas la personne adéquate. II craint que, vu son militantisme connu de tous, la manœuvre ne soit rapidement dénoncée. La situation devient d'autant plus compliquée lorsque Mikado, citoyen soviétique, est approché pour la même mission par l'ambassade d'U R SS. Mikado en fait la confidence à Hertz J ospa, qui tente de l'en dissuader et exige qu'il informe

\footnotetext{
${ }_{1}$ Cet article offre un aperçu d'une recherche présentée en juin 2006 dans le cadre d'un travail de DEA à I'ULB/CIERL-Institut d'études du judaïsme (I nstitut Martin Buber) : Arnaud Bozzini, $L$ a reconstruction de la sociabilité juive à B ruxelles (1944-1954). Le cas de la "Solidarité juive", ULB /CIER L-I nstitut Martin Buber, Bruxelles, 2006. N ous nous étions alors proposé d'exposer ce qu'a pu être l'entreprise de reconstruction de la vie juive au lendemain de la Libération dans un milieu donné qui, en raison d'un engagement de longue date, s'est vu occuper une place de choix dans les espoirs et les réalisations de l'immédiat après-guerre. Mais aussi de dégager des éléments de réflexion sur cette entité sociologique spécifique qu'est le militant juif communiste d'après 1945, pour lequel l'analyse des relations entre les J uifs communistes et le PCB était particulièrement révélatrice. Cette étude se poursuit actuellement dans le cadre d'une thèse de doctorat sous la direction de J ean-Philippe Schreiber au Centre interdisciplinaire d'É tude des R eligions et de la $L$ aïcité ( $C I E R L-U L B)$.

2 « N ote de B. Berger à R. Dispy », Commission centrale de Contrôle politique (CCP), n 0384, CAR COB.

3 P our un portrait de Chlioma F ridman, lire : J -Ph. Schreiber, Dictionnaire biographique des J uifs de $B$ el gique. F igures du judaï sme bel ge X I X e-X X e siècles, B ruxelles, 2002, p. 117.

4 H ertz J ospa (1905-1966), originaire de B essarabie. A cquis à la lutte des classes et au marxisme, il arrive en B elgique pour étudier au début des années 20. C'est alors qu'il adhère au PCB. II est l'un des initiateurs du Comité de défense des J uifs (CDJ ), dans lequel il joue un rôle de premier ordre jusqu'à son arrestation en juin 1943. C'est l'une des grandes figures juives bruxelloises de l'après-guerre. J usqu'à son décès en 1966, il occupe plusieurs postes à responsabilités dans les instances du PCB.
} 
l'ambassade de la confidence. Les Soviétiques demandent alors la discrétion de H ertz I ospa et attendent une décision du PCB quant à la mission que ce dernier souhaite confier à Mikado. La convalescence de H ertz J ospa, rescapé des camps, et la lenteur dans la transmission de ces demandes aboutissent à ce que Mikado, pressé par l'ambassade, passe chez les sionistes de gauche. S'ensuivent les articles de Mikado dans la presse du Poale T sion de gauche et les explications de H ertz J ospa devant les instances du Parti5.

L'affaire Mikado pourrait se réduire à une simple entreprise d'espionnage ratée si elle n'était pas dans son déroulement et ses consé quences - sur lesquelles nous reviendrons plus en détail par la suiterévélatrice des tensions entre la direction du PCB et ses militants juifs. Cette histoire rend particulièrement perceptible la radicalisation qui s'opère au sein de la direction du PCB, depuis la Libération, à l'égard de ce qu'il estime être un particularisme ethnique inacceptable.

N ous traiterons dans cet article de la progressive dégradation des rapports entre le PCB et "ses" J uifs. N ous avons pour cela exploité les nombreux rapports thématiques et personnels de la Commission de Contrôle politique du PCB conservés au Centre des Archives communistes en B elgique (CAR COB) ainsi que les archives de H ertz J ospa. $L$ 'analyse des rapports entre le PCB et ses militants juifs apporte un certain nombre d'éléments qui permettent de saisir la progressive marginalisation des communistes juifs dans la "rue juive" ${ }^{\prime \prime}$. De la chronologie avancée par J osé Gotovitch dans un article consacré à ce sujet, il ressort trois temps dans l'évolution des relations au sortir de la guerre : de la L ibération au printemps 1945, période durant laquelle on assiste à la tentative d'une affirmation juive spécifique au sein du PCB ; ensuite, et ce jusqu'au début de 1950, on constate une progressive dégradation des rapports entre le $\mathrm{PCB}$ et ses militants d'origine juive qui marginalise ces derniers dans la "rue juive" ; enfin, à partir de 1950, les décisions prises de la part du PCB marquent véritablement la rupture?.

\footnotetext{
5 II s'agit de la version de l'affaire telle qu'elle est relatée dans une note rédigée par $\mathrm{H}$. J ospa : «N ote J ospa concernant l'affaire F ridman », archives privées $H$ ertz et $Y$ vonne J ospa, $n^{\circ} 16$, s.d. ${ }^{6} \mathrm{~L}$ 'expression "rue juive" réfère à l'opinion publiquejuive.

7 J. Gotovitch, "J uif sur la pointe des pieds: le Parti communiste belge et les J uifs dans l'immédiat après-guerre. Notes succintes. », dans J.-Ph. Schreiber (éd.), H ertz J ospa. J uif, résistant, communiste, 1997, pp. 101-110.
} 


\section{La " Solidarité juive", une section juive du PCB ?}

À la Libération de Bruxelles, en septembre 1944, les militants juifs communistes occupent une place significative au sein de la "rue juive" bruxelloise. F ort de leurs engagements dans la R ésistance tant civile qu'armée, ils vont peser sur les enjeux et les espoirs de l'immédiat après-guerre. L'empathie qu'ils tirent de leur rôle pendant I'Occupation et le crédit dont bénéficie le $P$ arti communiste de B elgique (PCB) et I'Union soviétique leur permettent, dans les premiers mois qui suivent la Libération, de participer pleinement au redressement de la vie juive et de marquer de manière indélébile la collectivité juive bruxelloise.

Par le biais d'une organisation d'entraide, la "Solidarité juive" plus communément nommée la Sol -, les communistes juifs mettent en place des infrastructures qui assurent un travail social, culturel et pédagogiques. De la sorte, ces militants tentent d'abord de répondre à la situation d'urgence et ensuite d'offrir à la population juive bruxelloise les conditions d'une réinsertion?.

Si la Solidarité juive est un mouvement engagé dans la reconstruction de la vie juive, elle est aussi et avant tout une section juive du PCB, dont les options sont modelées en fonction des directives du Parti communiste alors au faîte de sa puissance. Cette organisation est, en cet automne 1944, une cellule active de communistes juifs liés à la direction fédérale du PCB et un cercle de "compagnons de route" re lativement politisés ${ }^{10}$. Dès lors, à l'activisme social de la Solidarité

\footnotetext{
8 La "Solidarité J uive" est vraisemblablement créée à l'automne 1939. Le pacte germanosoviétique ayant mis un terme aux pérégrinations du F ront populaire (qui agite la gauche belge depuis le milieu des années 30), on assiste à un repli de la famille communiste sur elle-même. Cette situation entraîne un nouvel isolement des militants juifs. Dès lors, des anciens des $P$ atronatn et du Comité juif pour l'E spagne vont mettre en place, en conformité avec les directives du PCB, une structure qui cherche à répondre aux exigences créées par la dégradation de la situation politique européenne. La naissance de "Solidarité J uive" répond à cet isolement. II s'agit d'un organisme de soutien aux militants et sympathisants juifs communistes en B elgique qui se veut être une structure d'accueil pour les réfugiés qui fuient I'Allemagne nazie. Voir R. Van Doorslaer, E nfants du ghetto. J uifs révoluti onnaires en B elgique ( 1925-1940) , B ruxelles, 1997, p. 208.

9 «R apport de l'activité de Solidarité juive pour 1944 et 1945 », dans Solidaritet, n 9, 7.12.1945, p. 1 (traduit du yiddish par B oris Szyster).

10 À cet égard, il est révélateur de consulter l'organigramme de la "Solidarité juive" pour cette période et d'y constater que la plupart des responsables au Comité, dans les sections locales et dans les commissions sont membres du PCB. «Organigramme de Solidarité juive », s.d., dossier
} 
juive, s'ajoute l'audience électorale - toutefois éphémère - du PCB qui devient en fait, aux législatives de 1946, le troisième parti de B elgique, ce qui l'amène à participer à cinq gouvernements d'union nationale jusqu'au printemps 1947.

Cette situation explique pourquoi la trajectoire des militants juifs communistes est intimement liée à l'évolution des rapports entre le PCB et la société belge dans son ensemble.

\section{Une affirmation identitaire de courte durée}

Dès septembre 1944, les militants communistes juifs vont tenter d'asseoir leurs spécificités au sein du PCB par la création d'une Fédération des J uifs polonais et d'une Amicale des Prisonniers politiques juifs. Conscients qu'ils ont combattu pour la même cause et qu'ils partagent avec les "masses laborieuses" du pays les mêmes revendications politiques et sociales, ils refusent de s'isoler de la vie publique belge. Ils participent donc des espoirs qui sont nés, dans ces milieux, durant I'Occupation. Cependant, ils ne peuvent faire abstraction de leur identité - cellelà même pour laquelle ils se retrouvent dans cette situation - et du milieu dont ils sont issus. Dès lors, ils ne peuvent pas concevoir que le Parti communiste ne prenne pas en considération un certain particularisme identitaire.

J usqu'au printemps 1945, le PCB tait I'agacement que suscitent chez ses cadres ces affirmations identitaires spécifiques. Une note datée de juin 1945 et émanant de J ean Terfve, alors secrétaire général du $P C B$, vient mettre un terme au silence relatif du Parti sur la question. S'adressant à Berthe Berger, il rapporte I'hostilité absolue du secrétariat du PCB à la création d'une F édération des J uifs polonais. II admet tout au plus la mise en place d'une commission spécifique chargée des revendications des militants juifs. L'objectif que s'est fixé le Parti à l'égard de militants juifs est énoncé clairement. II s'agit de « casser les redoutables tendances des copains juifs à se replier sur euxmêmes »11. Pour la direction du PCB, les souhaits "d'autonomie"

\footnotetext{
juif: affaires juives (CCC septembre à octobre 1947), dossier V, CCP/N\%010, CAR COB. A. L apiower, L ibres enfants du ghetto, B ruxelles, 1989, p. 23.

11 «N ote de J. Terfve à B. B erger, 6/6/1945», dossier juif : affaires juives (septembre à octobre 1947), dossier $\mathrm{V}, \mathrm{CCP} / \mathrm{N} \%$ 1010, CAR COB.
} 
manifestés par les militants juifs « emboîtent le pas et font le jeu des sionistes »12. Dès lors, il dénonce des comportements "nationalistes" en rupture avec la ligne du PCB et désigne ceux qui en sont responsables.

Cette stigmatisation entraîne immédiatement une réponse de B erthe B erger. Elle réfute les accusations portées contre ses camarades ainsi que toute collusion avec les sionistes ${ }^{13}$. Selon elle, il s'agit d'une perception "statique" donnant lieu à des analyses erronées qui ne tiennent compte ni de la situation complexe d'une communauté juive dévastée par la guerre ni de l'évolution du contexte historique et démographique en $\mathrm{P}$ alestine.

À ces tensions s'ajoutent celles nées de la création en octobre 1944 de I'Aide aux I sraélites Victimes de la Guerre (AIVG). Au lendemain de la Libération, pour satisfaire les demandes et répondre aux angoisses économiques, sociales et psychologiques, il apparaît indispensable d'organiser une représentation juive qui puisse se poser en interlocuteur légitime face aux autorités belges. La revendication d'un tel organisme apparaît comme la condition sine qua non du retour à la normalité. La détermination unanime d'unir les tendances de la judéité belge pour relever les défis que pose le redressement de la communauté juive va rapidement devenir l'un des enjeux de l'immédiat aprèsguerre. En effet, occuper une place de choix au sein d'un organisme central apparaît comme le moyen de peser sur les orientations prises pour assurer l'avenir de la communauté juive.

Le PCB perçoit d'un mauvais œil la création de I'AIVG en lieu et place du Comité de Défense des J uifs (CDJ ). La Solidarité juive, "section juive" du PCB qui jouit d'une audience significative dans la "rue juive" exige une place de choix au sein de I'AIVG. En tant qu'organisation "de masse", elle souhaite y retrouver la place qui était la sienne durant l'Occupation. Les communistes comprennent que cette nouvelle redistribution des cartes s'inscrit dans le cadre des orientations politiques belges globales qui visent à évincer les communistes du pouvoir. Ils prennent conscience du danger constitué par une association qu'ils ne contrôlent pas et qui risque de les

\footnotetext{
$12 \mathrm{~N}$ ote rédigée par $\mathrm{J}$. Grippa au secrétariat du Parti concernant la politique à mener parmi les J uifs, 9.10.1945, dossier juif : affaires juives (septembre à octobre 1947), dossier V, CCP $/ \mathrm{N} \% 010$, CAR COB.

13 « N ote de B. B erger à R. Dispy, 15.10.1945», dossier juif : affaires juives (septembre à octobre 1947), dossier $\mathrm{V}, \mathrm{CCP} / \mathrm{N} \%$ 010, CAR COB.
} 
marginaliser. Alors que les premiers jalons de la reconstruction sont posés, ils risquent de ne pouvoir maintenir et entretenir le capital de sympathie accumulé durant l'Occupation.

\section{Hertz J ospa, l'homme providentiel ?}

Les protestations argumentées de Berthe Berger n'enrayent nullement la dynamique initiée au printemps 1945 par J ean Terfve. Les accusations de « déviations à caractère chauvin » que traduirait la volonté de doubler les organisations existantes de groupements juifs, se multiplient. Elles se portent sur la personne de Richard W olman ${ }^{14}$. Pour J ean Terfve, le temps est venu de "procéder à un solide nettoyage et à une mise au point $\$^{15}$. S'il est vrai que la polémique tourne autour des revendications juives, elle se concentre essentiellement sur des responsables de la Solidarité juive membres du $P C B$, qui jouissent d'une écoute au sein du milieu concerné et ont joué, comme nous le verrons par la suite, un rôle dans la R ésistance. À la veille de 1946, le malaise est grand et les relations entre la direction du Parti communiste et "ses" militants juifs sont tendues. Le Parti va opter pour une négation de la problématique, bientôt réduite à des dénonciations de personnes pour attitude autoritaire ou comportements nationalistes, voire pour activisme sioniste. La réorganisation du travail juif afin de mettre un terme aux velléités identitaires est alors décidée. Le P arti nomme H ertz J ospa responsable national du travail juif et secrétaire politique de la section des militants du PCB de langue yiddish. II est chargé de mener en milieu juif un travail plus efficace et plus conforme à la ligne du PCB. À l'époque, selon Maxime Steinberg, Hertz J ospa rédige une note intitulée $L$ a question juive en $B$ elgique, qui porte sur la complexité des relations judéo-communistes et les orientations politiques des J uifs de

\footnotetext{
14 Moniek Wolman (1906-1999), dit «R ichard ». Originaire de Pologne, il arrive en B elgique en 1933. R esponsable de la section juive du Secours rouge international, il participe à la création de la "Solidarité J uive" en 1939. Actif dans la Résistance, il devient, dès septembre 1944, le secrétaire de la "Sol". Au cours de cette période, il connaît de nombreux déboires avec le PCB, dont il est membre.

15 « N ote de J. Terfve à Clavel, L almand et Dispy, 10.12.1945», dossier juif : affaires juives (CCC septembre à octobre 1947), dossier V, CCP/N $\% 1010$, CA R COB .
} 
B elgique à la veille de la guerre ${ }^{16}$. I I n'est toutefois pas inconcevable de projeter, dans une certaine mesure, cette situation au lendemain de la Libération. L'Occupation nous apparaît comme une parenthèse qui a évacué, en partie et pour quatre années, les tensions nées de la politique assimilationniste du PCB et des revendications identitaires juives. Dès lors, il est probable qu'à sa nomination, à l'automne 1945, H ertz J ospa a trouvé une situation idéologique (sur laquelle venait bien évidemment se greffer le poids de l'occupation) comparable à celle qu'il décrit. Pour lui, le problème réside dans le manque de cadres, dans l'insuffisance du travail en milieu juif et dans le «sectarisme [... ] de nombreux camarades juifs ». Cette situation est due, en partie, au fait que tout militant (juif) compétent est systématiquement assigné à une fonction en dehors de la "rue juive".

II est évident, et ce depuis l'entre-deux-guerres, que le PCB refuse de poser le problème des militants juifs en termes socio-culturels. Le phénomène n'est appréhendé que d'un point de vue économique. C'est pourquoi, face à des revendications dont le PCB ne veut saisir le sens, il adopte une politique qui vise à évacuer le problème avec pour conduite le transfert des cadres juifs dans la "rue belge", la mise au ban du Parti de certains de ses militants juifs pour activisme nationaliste et, en décembre 1945, la nomination d'un homme fort de son passé de résistant, acquis au PCB et ayant l'écoute des milieux juifs.

\section{L'affaire Mikado}

Tandis que l'arrivée de Hertz J ospa avait pour objectif de « renverser la vapeur » et de redresser les erreurs de R ichard W olman, accusé de complaisance à l'égard des sionistes, la confrontation devient directe entre le Parti communiste et "ses" J uifs"17. A lors que l'incompré hension marque les rapports judéo-communistes, l'affaire Mikado, elle, vient relancer la polémique.

Ce qui nous paraît intéressant dans l'affaire Mikado, au-delà d'un comportement schizophrénique entre la direction du PCB, les

\footnotetext{
16 H ertz J ospa, «La question juive en B elgique » (introduction de Maxime Steinberg), dans J .Ph. Schreiber (éd.), H ertz J ospa. J uif..., 1997, p. 143.

17 « J ospa H (g)ertz », Commission centrale de Contrôle politique (CCP ), n 2413, CAR COB .
} 
responsables de sa "section juive" et les Soviétiques, c'est l'instrumentalisation qui en est faite par le PCB ${ }^{18}$.

La procédure disciplinaire à laquelle cette affaire aboutit, vise à mettre un terme aux « déviations nationalistes des camarades juifs ». Passé au P oale T sion de gauche au printemps 1947, Mikado signe des articles qui sont à l'origine de la polémique ${ }^{19}$. Pour Mikado, bien qu'il soit probable que ce soit $\mathrm{H}$ ertz J ospa qui ait rédigé le texte, c'est donc à la «lumière des événements récents » qu'il convient d'examiner la situation. II va sans dire que "la catastrophe hitlérienne» a mis en connexion la «question juive» et celle de la Palestine, et ce, même pour les « asionistes » et les progressistes. Cette situation impose, sel on l'auteur, de soutenir la création d'un «foyer national et culturel en Palestine ». Cette issue « imparfaite» n'est cependant que provisoire, la résolution définitive du « problème juif » demeure pour le rédacteur l'avènement du socialisme.

Ce passage ne sera nullement perçu par la direction du Parti comme une entreprise d'espionnage téléguidée par les Soviétiques. L 'échec de l'opération, puisque l'ostracisme dont Mikado est progressivement victime l'éloigne de ses anciens camarades au profit des rangs sionistes, est perçu comme une trahison qu'ont tenté de couvrir B erthe B erger et H ertz J ospa20. De cette histoire, la direction du PCB ne retient que les silences des responsables du travail juif et la participation de $\mathrm{Hertz}$ J ospa aux écrits de Mikado. Rien n'est dit sur l'acceptation de la proposition de Berthe Berger en avril 1946, ni sur la note faite à Raymond Dispy, une des personnalités marquantes du Parti, qui l'informait du passage de Mikado au P oale T sion de gauche21. Quant à la responsabilité de l'ambassade soviétique dans la rédaction des articles, elle n'est que timidement évoquée22. P our le PCB , l'important n'est pas là. Les éléments qui doivent permettre de remettre au pas les J uifs communistes sont réunis.

\footnotetext{
18 J. Gotovitch, op. cit. , pp. 107-108.

19 M. F ridman, «Le chemin de la vérité», dans U ndzer V ort, $n^{\circ} 16,18.4 .1947$, p. 4 (traduit du yiddish par J oseph Szyster).

20 Sh. Fridman, «Si je m'étais appelé Gromyko», dans U ndzer V ort, $n^{\circ} 26,27.6 .1947$, p. 2 (traduit du yiddish par J oseph Szyster).

21 «Fridman Chlioma/Mikado», Commission centrale de Contrôle politique (CCP), $n^{\circ} 0885$, CAR COB.

22 | bid.
} 
A lors que l'affaire Mikado alimente les tensions, le Parti organise, après avoir dissous "l'A micale juive», un grand coup de filet dans la "rue juive". À cette polémique en succèdent d'autres, qui mettent en cause des responsables juifs membres du Parti et de la Sol. Le PCB exige la fin de toute affirmation identitaire et refuse de poser la "question juive".

\section{Le temps des affaires}

À I'automne 194723, Richard Wolman - alors secrétaire à la Solidarité juive - et J acob Gutfrajnd ${ }^{24}$ sont mis en cause. Ils sont accusés d'être des indisciplinés ambitieux, manipulateurs et arrivistes qui agissent avant tout, non dans l'intérêt du Parti, mais pour leur compte personnel25. Outre cette mise en cause de leur comportement, ils sont également taxés, et ce avec un troisième "larron", Ber Szyster, de gestion douteuse voire d'irrégularités financières dans le traitement des "pages belges" de la $\mathrm{N}$ aïe $\mathrm{P}$ resse de $\mathrm{P}$ aris'26.

Mais ce qui aggrave leur situation, du moins pour J acob Gutfrajnd et $R$ ichard Wolman, et qui apparaît de manière patente ou en filigrane dans les archives, ce sont leurs "déviations politiques". L'opposition à la suppression de l'Amicale, la volonté sinon de structures indépendantes, du moins de sections juives à l'intérieur des organes du

\footnotetext{
${ }^{23}$ L es premiers documents dont nous disposons à propos de ces « affaires » commencent à cette époque (cfr. dossier juif : affaires juives (CCC septembre à octobre 1947), dossier V, CCP $/ \mathrm{N} \% 010$, CAR COB).

24 J acob Gutfraynd (1909-1991), dit «Albin ». Originaire de Pologne, il immigre en B elgique puis en France à la fin des années 30. Revenu à Bruxelles, il participe à I'organisation du premier groupe de partisans juifs avant de devenir commandant de la P remière Compagnie juive du Corps mobile de Bruxelles des Partisans armés. Membre du PCB, il est actif à la Solidarité juive et rédige de nombreux articles dans U nzer $\mathrm{K}$ amf et Solidaritet. Après son exclusion du $\mathrm{PCB}$, il retourne en Pologne en 1952. D éçu de cette confrontation avec le "socialisme réel", il immigre en I sraël en 1957.

25 «Bureau pour le travail juif, rapports concernant les camarades J acob (Albin), Richard et B oris (Bruxelles, 20.9.1947) », dossier juif : affaires juives (CCC septembre à octobre 1947), dossier V, CCP $/ \mathrm{N} \% 010$, CAR COB.

${ }^{26}$ Ber Szyster (1907-1989), dit «B oris ». Originaire de Vilna, il arrive en B elgique en 1929 pour y faire des études à l'université de Gand. II s'établit par la suite à Bruxelles où, proche de la mouvance communiste, il finit par adhérer au PCB. En 1939, il est l'un des fondateurs de la Solidarité juive et intègre la R ésistance sous l'Occupation. II sera d'ailleurs le rédacteur des numéros clandestins de $\mathrm{U}$ nzer $\mathrm{K}$ amf. Au lendemain de la L ibération, il est une des figures actives de "Solidarité J uive" et continue à rédiger de nombreux articles pour la presse yiddishophone d'obédience communiste (U nzer $\mathrm{K}$ amf, Solidari tet et les pages belges de la $\mathrm{N}$ aïe $\mathrm{P}$ resse).
} 
Parti et la mise en cause de la clairvoyance du Comité central quant au traitement des militants juifs, traduit, pour le Parti, un « état d'esprit sectaire et indiscipliné »27. Or, selon le PCB, ces protagonistes sont progressivement devenus incontournables dans la "rue juive". Parlant de J acob Gutfrajnd, un rapport constate que « beaucoup de camarades vont chez lui pour recevoir des conseils et des directives» au lieu de s'adresser au Comitéz8. Cette situation est, bien entendu, inacceptable pour le Parti, puisque R ichard Wolman comme J acob Gutfrajnd ne tiennent pas compte de la ligne du bureau. Le PCB, qui impute la dégradation de la situation à leurs agissements, va prendre des sanctions à leur égard.

Cette confrontation, qui vise à mettre un terme à toute affirmation juive, ne se fera pas sans quelques remous. Au terme de "I'enquête" menée par le Parti, les sanctions sont lourdes. La Commission de Contrôle politique décide d'exclure J acob Gutfrajnd, alors qu'elle prononce un blâme à l'encontre de Ber Szyster et de R ichard W olman.

E n novembre 1947, lors de l'assemblée générale des camarades juifs, les différents verdicts de la Commission de Contrôle politique sont prononcés 29 . On assiste à un règlement de compte entre le PCB et "ses" militants juifs un peu trop "turbulents". En effet, les sanctions prises dans le cadre des "affaires" portent un coup sérieux aux militants communistes de la "rue juive" et particulièrement aux cadres de la Sol.

$L$ 'indignation est grande. A u cours de cette réunion, la contestation se fait entendre. L'exclusion de J acob Gutfrajnd notamment est remise en cause. De l'opposition à la suppression, vieille de deux ans, de l'A micale aux irrégularités financières qui sont vraisemblablement des problèmes de mauvaise gestion, ils sont beaucoup à considérer que ce sont des motifs insuffisants pour l'exclure.

À la lecture des archives portant sur cet épisode, on perçoit le choc et l'incompréhension qui règne alors chez les militants juifs. On a été jusqu'à mettre en cause l'attitude de certains camarades durant l'Oc-

\footnotetext{
27 «Bureau pour le travail juif, rapport concernant le camarade J acob-A Ibin, (Bruxelles, 20.9.1947) », dossier juif : affaires juives (CCC septembre à octobre 1947), dossier V, CCP $/ \mathrm{N}^{\circ} / 010$, CAR COB.

28 «R apport sur les camarades Richard, J acob et Boris (Bruxelles, 28.9.1947) », dossier juif : affaires juives (CCC septembre à octobre 1947), dossier V, CCP/N $\% 10$, CAR COB.

29 «P rotocole de l'assemblée générale des camarades juifs (12.11.1947) », dossier juif : affaires juives (CCC septembre à octobre 1947), dossier V, CCP/N $\% 10$, CA R COB .
} 
cupation, alors que le rôle joué par les résistants juifs communistes communistes juifs (nous éviterons ce débat historiographique) - occupe une place centrale dans le discours des J uifs communistes. On ne s'est pas limité aux "agitateurs", à des personnalités qui, il est vrai, ne devaient pas faire l'unanimité. On s'en est même pris à H ertz J ospa, c'est-à-dire à celui qui avait été rappelé pour mettre de l'ordre et qui semblait avoir les faveurs du Parti. Une des clés de compréhension, comme nous tenterons de le montrer dans nos prochaines recherches, réside dans cette attaque en règle à l'égard d'anciens résistants. II est probable que, dans l'immédiat après-guerre, la direction du PCB ait voulu régler une série de conflits nés durant l'Occupation ou antérieurs à 1940.

\section{À deux pas de la rupture}

L'émoi est loin d'être anodin. Le propos tenu par les J uifs communistes à l'égard de la R ésistance, s'il s'inscrit dans un discours patriotique et national, n'en élude pas pour autant la dimension juive. La "R ésistance juive", qu'on ne définit pas, est l'élément constitutif de ce point de vue au sortir de la guerre. Cette absence de définition est explicite quant à la perception qu'ils ont d'eux-mêmes. À quoi peut bien servir d'expliquer ce qui leur paraît une évidence, à savoir une perception identitaire qui leur est toutefois déniée par le PCB. Si les propos patriotiques tenus sont caractéristiques du vocable propre à ce type d'associations au lendemain du conflit, ce qui nous surprend dans le cas présent, c'est l'insistance portée à la dimension juive de la démarche. Le contenu du discours devient un référent identitaire. Dans le propos tenu par les J uifs communistes de la Sol, la "R ésistance juive" n'est ni gommée par la Résistance nationale, ni, par la suite, dépassée par les victoires militaires du jeune État d'I sraël30. Cette dimension "ethnique" n'est pas occultée par la mémoire du génocide31. C'est la figure du partisan - et plus encore celle du partisan armé - et non la thématique de la "passivité juive" qui prévaut. II y a une

\footnotetext{
30 Nous faisons ici référence au "diptyque" mis en exergue par Maxime Steinberg (voir $M$. Steinberg, «Problématique de la R ésistance juive en B elgique», dans $P$ oints critiques, $n^{\circ} 2,1979$, pp. 8-29.

31 M. Steinberg dans la préface à P. B roder, D es J uifs debout contre le nazi sme, B ruxelles, 1994, p. 19.
} 
absence flagrante - mais qui est loin d'être propre à ce milieu - de l'image du déporté. Cette absence est d'autant plus aisée que l'histoire des J uifs communistes sous l'Occupation est intimement liée à la $R$ ésistance.

Au sortir de la guerre, alors que les mémoires de la persécution sont modelées sur l'idée du martyre national, l'image qui s'impose en milieu judéo-communiste comme à l'ensemble de la société belge est celle du héros de la Résistance, victime de la répression nazie. Sous la pression d'une tradition patriotique qui honore exclusivement ses combattants et d'une tradition "antifasciste" inclusive, les victimes juives de la persécution raciale sont assimilées sinon à des antifascistes, du moins, à des victimes disparues pour la grande cause 32.

La "R ésistance" est donc une thématique omniprésente chez les J uifs communistes. Elle remplit une fonction identitaire au contenu émotionnel fort. Assumer le choix de l'assimilation - ce à quoi le Parti les presse - est une démarche qui implique des renoncements conséquents. Dès lors, il est probable que le milieu juif progressiste des années 40 et 50 ait surinvesti ce passé récent afin de pouvoir, sans pour autant renoncer à son engagement universaliste, transmettre une dimension juive. $L$ a mémoire de la $R$ ésistance devient le vecteur qui permet en milieu judéo-communiste de transmettre une forme de judéité. Comme le démontre Alain Lapiower, se faire l'héritier de la R ésistance juive - ou plus simplement de la participation de J uifs à la R ésistance -, permet d'assumer la seule part de judéité qui s'impose à l'évidence dans ce milieu et qui n'entre en rien en conflit avec la ligne du PCB 33 .

À la veille de ce règlement de compte, la direction du PCB était consciente des actes qu'elle allait poser et des ruptures que cela pourrait éventuellement produire. Le souci de mesure manifesté par J ean Terfve en est une preuve, lui qui, quelques mois auparavant, se proposait de « renverser la vapeur $»^{34}$. Toutefois, en cet automne 1947, I'heure n'est plus au pragmatisme avec les revendications juives. L a situation ne l'exige d'ailleurs pas. Soit ces militants se cantonnent dans

\footnotetext{
32 P. L agrou, M émoires patri otiques et occupation nazie, B ruxelles, 2003, p. 239.

$33 \mathrm{~A}$. L apiower, «H ertz J ospa et le milieu juif progressiste. Une légende vivante», dans J .-Ph. Schreiber (éd.), H ertz J ospa. J uif, résistant, communiste, B ruxelles, 1997, pp. 120-122.

34 «J ospa H (g)ertz », Commission centrale de Contrôle politique (CCP), n² 2413, CA R COB.
} 
leurs attitudes nationalistes et quittent le Parti ; soit ils retournent en Pologne et adhèrent au $P$ arti communiste polonais; soit ils intègrent pleinement, au sein du PCB, la "rue belge". Les alternatives sont clairement formulées, la rupture eût été imminente si une nouvelle parenthèse n'était venue offrir un nouveau répit.

\section{La guerre d'Indépendance d'Israël, un dernier répit}

Au printemps 1948, I'engouement pour la guerre d'I ndépendance $d^{\prime}$ I sraël apaise, pour un temps, les tensions entre les J uifs communistes et la direction du Parti. À vrai dire, la reconnaissance par l'URSS, dans la nuit du 14 au 15 mai, de l'État d'I sraël, accompagnée de la déclaration prosioniste du représentant soviétique à I'ONU, Andreï Gromyko, a de quoi surprendre. Elle enthousiasme les J uifs communistes, qui y voient aussi une reconnaissance de leur identité par le Parti. La compréhension opportuniste exprimée à l'égard d'une affirmation nationale juive va accorder un bref répit à la confrontation entre les communistes juifs et le Parti. À la veille de la guerre d'Indépendance, l'attitude des J uifs communistes à l'égard de la "question palestinienne" est conciliante et ouverte ${ }^{35}$. Le deuxième conflit mondial a incontestablement changé la donne. Bien que l'exigence demeure une progressive intégration à la société belge et que le sionisme est toujours fustigé comme une attitude nationaliste, la création d'un É tat juif en Palestine n'est plus rejetée en bloc comme durant les années 30. On comprend dès lors, au vu des positions que nous venons d'exposer et d'un PCB peu enclin aux concessions face à ses militants juifs, la surprise provoquée par la déclaration de Gromyko. Alors que le désaveu de toute revendication identitaire ne date pas d'une année, la situation met rapidement en évidence les contradictions au sein du Parti. Toutefois, au regard de la "question juive", le PCB n'en est pas à sa première volte-face. Assuré du soutien quasi inconditionnel des militants juifs à la cause communiste, la contradiction est assumée.

35 «J ospa H (g)ertz», Commission centrale de Contrôle politique (CCP), $n^{\circ} 2413$, CAR COB, et réflexions de J. Gold sur un article publié dans L 'appel des jeunes (antérieur à mai 1948), dossier contacts avec des $\mathrm{PC}$ par rapport à des questions de personnes, classeur Pologne, dossier $\mathrm{V}$, $\mathrm{CCP} / \mathrm{N} \% 008$, CAR COB. 
À la Sol, les militants sont partagés entre le scepticisme, voire la désapprobation pragmatique des anciens, et l'enthousiasme des jeunes, très réceptifs aux événements. D'autant que la constitution d'un contingent de volontaires de I'U nion sportive des jeunes J uifs (USJ J ) pour la Palestine pose à nouveau le problème déjà soulevé par $\mathrm{H}$ ertz J ospa quant à l'absence de cadres. L'USJ J , initiative des plus jeunes membres de la Sol qui voit le jour dès l'automne 1944, est en quelque sorte une projection vers l'avenir des espoirs que la Sol met dans la reconstruction. Ces jeunes incarnent non seulement l'avenir du "peuple juif" mais également le futur du mouvement. Au-delà de la réinsertion sociale, I'USJ J , projection des aspirations et des comportements de la Sol, affiche clairement son engagement politique et sa volonté d'incarner l'avenir. Or, dans le cas présent, cet engagement permet aux jeunes de I'USJ J de conjuguer la logique politique et leur attachement au judaïsme. II s'agit de se battre pour une terre juive et socialiste, sous couvert non de sionisme mais de lutte anticolonialiste. L'enthousiasme de ces jeunes gens s'inscrit également dans une démarche d'identification. Pétris par la mythologie communiste de la guerre d'E spagne et de la R ésistance, leur éventuelle participation à la guerre d'Indépendance les identifie à cette lignée de héros communistes véhiculée par leur mémoire collective.

Au cours de cette brève période, mis à part les doutes émis par certains dirigeants de la Sol, la situation est relativement commode. La direction stalinienne du Parti et ses militants juifs sont pour une ultime fois en accord.

$L$ 'initiative de constituer un groupe de volontaires issus des rangs de l'USJ J n'est pas sans interrogation. Les différents fonds d'archives et les témoignages ne permettent pas de répondre de manière tranchée à la question. Qui est à l'origine de la "suggestion" ou de "I'ordre" ? L'idée vient-elle de l'USJ J qui en aurait référé au Parti afin d'obtenir son approbation ? Où est-ce le Parti qui a "suggéré" cette décision, sachant qu'il disposait de jeunes prêts à s'investir dans ce projet et dont «l'attachement inconditionnel [au Parti] était une réalité»?36 Dans cette histoire, les témoignages des volontaires divergent et les volteface du Parti n'aident en rien à éclaircir le propos.

36 Témoignage de M. Dorn recueilli par A. Lapiower, cité dans A. Lapiower, Libres enfants du ghetto, 1989, p. 55. 
Pour David Susskind37, I'un des fondateurs de l'USJ J , I'idée d'envoyer des volontaires combattre en I sraël est née dans les rangs de I'USJ J ${ }^{38}$. II en a alors fait part à $\mathrm{H}$ ertz I ospa, mais vu les réticences de ce dernier, il s'est adressé à la direction du PCB. Quant à Victor Cygielman39, également à l'origine de la création de l'U nion sportive juive, il affirme que la décision a été prise par le comité de I'USJ J ${ }^{40}$. Lorsque le militant communiste J érôme Grynpas quitte la Belgique pour participer à la Guerre d'I ndépendance de l'É tat hébreu, il est en possession d'une lettre de recommandation du PCB pour son homologue israélien ${ }^{41}$. Le Parti communiste n'est donc pas tout à fait extérieur à cette décision puisque, s'il n'est pas l'instigateur du mouvement, il le cautionne. Au regard des tensions entre le Parti, la Sol et son mouvement de jeunesse, on imagine mal un PCB en retrait. La direction du PCB n'a certainement pas ordonné, ce n'est pas comme cela qu'elle agit. E lle a peut-être au plus souhaité instamment cette participation. Mais ce qui est clair, c'est que le $P C B$, en autorisant ses militants au départ, a avalisé cette décision.

Outre un alignement sur la politique de Moscou, quelles sont les motivations qui expliquent l'aval donné par le Parti ? Le PCB a pu

\footnotetext{
37 David Susskind (1925). Né à Anvers dans une famille religieuse, c'est au cours de l'occupation et au contact de la Résistance qu'il se rapproche du PCB. A la Libération, il est l'un des fondateurs de I'USJ J. Proche du Bureau politique, il quitte le PCB prosoviétique pour la dissidence chinoise de J acques Grippa en 1964. I I en sera cependant exclu pour « sionisme ». C'est alors qu'il se lance dans la création et l'animation du Cercle culturel et sportif juif (CCSJ ) - Centre culturel laïc juif (CCLJ), dont il est aujourd'hui le président honoraire.

$38 \mathrm{~V}$. Engel, Le don de M ala-Léa. David Susskind: I'itinéraire d'un M ensch, Bruxelles, 2006, pp. 161-181.

39 Victor Cygielman (1926-2007). Originaire de P ologne, sa famille arrive au début des années 30 en Belgique. C'est au cours de l'occupation et en réaction au refus de I'H ashomer $\mathrm{H}$ atzaïr de participer à la résistance aux nazis qu'il se rapproche du FI. À la Libération, il participe à la fondation de I'USJ J , dont il assume avec David Susskind la "direction". II est d'ailleurs I'un de ceux qui participe, sous la "bannière" USJ J à la guerre d'I ndépendance d'I sraël. I I part construire le socialisme en Pologne en 1952. Cette confrontation l'éloigne définitivement du communisme. II vivait depuis 1957 en I sraël.

40 Correspondance entre Victor Cygielman et J oseph Szyster, papiers personnels de J oseph Szyster, mars 2006.

41 J érome Grynpas (1929), issu d'une famille d'origine polonaise, adhère au PCB à la fin de 1944. II entre à l'USJ J au lendemain de la Libération. C'est dans ce cadre qu'il part clandestinement, en mai 1948, en I sraël et participe à la guerre d'I ndépendance. II y demeure en 1949 et, lors des élections, milite dans la cellule communiste belge du Palmakh, unité d'élite de la $\mathrm{H}$ agana. De retour en B elgique où il entame des études d'abord à L ouvain puis à l'UL B (1953-1959), il est très actif au sein des É tudiants communistes, dont il occupe le secrétariat jusqu'en 1961.
} 
considérer qu'il s'agissait là d'une opération intéressante en termes de régulation interne. L'opposition de H ertz J ospa n'est-elle pas motivée par la crainte de voir I'USJ J , vidée de ses forces vives, péricliter ? Or, ce sont elles qui ont en partie mené la contestation au sein du PCB au moment des "affaires" de 1947.

Les archives communistes, quant à elles, nous offrent une autre version des faits. II faut cependant noter que les documents auxquels nous nous référons sont tardifs et interviennent dans un tout autre contexte. II s'agit d'informations relatives au contingent de I'USJ J et transmises aux communistes polonais. II n'est plus alors de bon ton de montrer que les communistes ont été un jour favorables à I sraël. Sel on ces documents, si ces jeunes ont reçu l'accord du PCB, ce n'est nullement ce dernier qui les a chargés d'une mission quel conque ${ }^{42}$. U ne note antérieure précise que «les camarades ne se rendaient pas en Palestine [pour le Parti] »43. Si le PCB n'en est pas l'instigateur, au vu de son attitude récente à l'égard de ses militants juifs, il a néanmoins approuvé l'initiative.

A vec l'intensification de la Guerre froide à la veille des années 50, on assiste à un revirement de la politique des communistes à l'égard d'Israël, qui a clairement opté pour le camp occidental. C'est à nouveau l'incompréhension qui se fait jour dans les rangs judéo-communistes. Les volontaires qui ont participé au contingent et qui reviennent se sentent lâchés, désavoués par le Parti. C'est par l'amertume que se solde l'expérience palestinienne. Surchargée émotionnellement, puisqu'elle liait la culture dont ces jeunes sont pétris à l'idéal politique dont ils se revendiquent, le désaveu de leur participation à la guerre d'I ndépendance leur laisse un goût amer.

\section{Construire le socialisme à l'E st !}

Un sentiment comparable sera d'ailleurs exprimé par ceux qui participent à l'aventure polonaise. De 1949 à 1954, un certain nombre de

\footnotetext{
42 «Lettre adressée par J. Borremans au Comité central du Parti ouvrier de Pologne (26.2.1952) », dossier contacts avec des P.C. étrangers par rapport à des questions de personnes, classeur Palestine, dossier $\mathrm{V}, \mathrm{CCP} / \mathrm{N} \% 008, \mathrm{CAR}$ COB .

43 «Note manuscrite d'un auteur non identifié (26.1.1952)», dossier contacts avec des PC étrangers par rapport à des questions de personnes, classeur Palestine, dossier $\mathrm{V}, \mathrm{CCP} / \mathrm{N} \% 008$, CAR COB.
} 
militants communistes juifs vont partir pour la Pologne. Motivés par les conditions de travail ou d'études qui leur sont proposées, ils se rendent dans le pays qui les a vus naître, eux ou leurs parents, pour participer à l'édification du socialisme. Les raisons de ces départs sont diverses et variées, elles finiront même par faire l'objet d'une note interne au PCB qui définit les cas dans lesquels la Commission de Contrôle peut accorder l'attestation pour le départ ${ }^{44}$. À la lecture de ce document, on constate que certains militants ont été "invités" par le Parti de gagner la Pologne. La "question juive", comme la nommait Hertz J ospa à son retour des camps, que le PCB refuse de poser devient incontestablement, aux yeux des dirigeants communistes, une réalité encombrante. II s'agit donc, pour le PCB, d'agir efficacement soit en évacuant cette dimension identitaire soit en intégrant ces J uifs à la "rue belge". N ous retiendrons de cette expérience que les militants doivent recevoir l'approbation du Parti communiste pour partir et que cette autorisation pour les cas dont nous avons pu prendre connaissance est conditionnée par l'accord de Hertz J ospa. Cette émigration est révélatrice de l'engouement pour le communisme en tant que facteur d'émancipation. Ces militants mettent une foi inconditionnelle dans le proj et socialiste tel que défendu par I'UR SS et les démocraties populaires d'E urope centrale. Les Soviétiques et leurs alliés ne sont pas seulement les libérateurs des camps, ils sont surtout ceux qui, en proposant une solution adéquate aux problèmes des nationalités, ont résolu la "question juive". La déception sera à la mesure de leurs espérances. Ils reviendront dépités et amers de Pologne. À leur retour, ils devront de surcroît faire face aux attaques de ceux qui, restés en Belgique, les accusent d'avoir renoncé et trahi leurs idéaux.

\section{La remise en cause d'un idéal}

E n ce début des années 50, les pressions exercées par la direction du PCB vont fortement peser sur les choix opérés. Dans le chef des

\footnotetext{
44 « N ote manuscrite relative aux attestations pour la Pologne (10.2.1951)», dossier juif : contact avec les PC étrangers par rapport à des questions de personnes-Pologne, dossier $V, C C P / N \% 008$, CARCOB.
} 
dirigeants communistes belges, il est évident que les militants juifs communistes vont s'intégrer à la "rue belge".

Depuis 1946, toutes les décisions prises par le PCB à l'égard des communistes juifs vont dans le sens d'une progressive assimilation. Le Parti, qui se refuse à toute prise en considération des aspirations identitaires juives, n'hésite pas à prendre des mesures qui visent à déstabiliser toute revendication en ce sens. Des condamnations de I'automne 1947 aux départs pour la Pologne, tout est mis en œuvre pour évincer ceux de ses militants qui revendiqueraient leur judéité. Au-delà du refus de poser clairement le "fait juif" au sein du PCB et d'une lecture marxisteléniniste de la "question juive" qui n'envisage que l'assimilation comme réponse à cette problématique, on perçoit une crainte au sein de la direction communiste. $\mathrm{E} n$ effet, si son attitude a un fondement idéologique, un autre aspect doit être pris en considération. Le PCB perçoit dans cette revendication en général et dans cette intention d'imposer des sections juives en particulier une volonté de se soustraire à son contrôle ${ }^{45}$. V $\mathrm{u}$ le mode de fonctionnement du PCB, une telle attitude ne peut être admise. Entre pur fantasme et paranoïa, c'est un Parti en perte de vitesse qui décide de régler ses comptes avec des militants en dehors de la ligne.

À la fin de l'année 1950, le PCB orchestre un véritable assaut contre la "rue juive". À la vue de certaines évolutions, le Parti va estimer que la "rue juive" n'a plus de raison d'être. Le dynamisme des militants juifs, notamment des jeunes, est nécessaire pour enrayer la décrue que le PCB a entamée dans la "rue belge"46. II est donc nécessaire de les y intégrer.

Dans un premier temps, on opère un rapprochement entre I'USJ J et la J eunesse populaire de B elgique (J PB) qui modifie la nature du mouvement de jeunesse juif. À l'automne 1950, le processus de collaboration entre les deux organisations s'accélère, puisque les sections de plus de 16 ans de l'USJ J sont dissoutes et ses membres

\footnotetext{
45 «R apport sur les camarades Albin, B oris et R ichard (8.10.1947) », dossier juif : affaires juives (CCC septembre à octobre 1947), dossier V, CCP/N \%010, CA R COB .

46 «B esoins d'effectifs jeunes », lettre du camarade De Munter (section Saint-Gilles), L e problème de la jeunesse, 10.12.1954, archives privées H ertz et $Y$ vonne J ospa, $n^{\circ} 14$. J . Gotovitch, op. cit., 1997, p. 109.
} 
"invités" par la direction du PCB, comme l'a démontré Alain L apiower, à rejoindre les sections local es de la J PB 47 .

À I'USJ J , le malaise est grand et ils sont peu nombreux à répondre à l'invitation qui leur a été faite. L'échec de cette politique d'intégration débouchera sur un compromis tacite et probablement perçu par la direction du PCB comme provisoire, à savoir la création d'une section juive au sein de la J PB. Ce groupe, appelé la section "Unité", regroupera de vingt à quarante jeunes, et ce jusqu'à sa dissolution par décision de la Commission de Contrôle du Parti à l'été 195248 .

La raison officielle avancée par le Parti communiste pour justifier cette décision est la suivante : ces adolescents ayant acquis, dans leur grande majorité, la nationalité belge, la nécessité d'une couverture légale par le biais d'une organisation sportive n'a plus de sens. D'autant, comme en atteste un rapport de la Sûreté de l'É tat, que cette organisation ne leurre pas les autorités belges qui sont conscientes de I'activisme politique procommuniste qui se déroule à I'USJ J 49. Cette dissolution, qui intervient au plus fort de la Guerre froide, nous semble davantage trouver ses motivations dans le non-sens que constitue pour le Parti communiste cette affirmation identitaire. Sans compter que cette décision alimente une J PB moribonde en éléments connus pour leur militantisme.

La disparition de I'USJ J est reçue comme un choc par les jeunes J uifs communistes. Ce mouvement, qui avait été au sortir de la guerre le lieu où ils avaient progressivement recréé une sociabilité, jouait un rôle essentiel dans leur vie. L'incompréhension ne vient pas d'un quelconque repli communautaire, comme tente de le dénoncer le PCB. Ces jeunes ne peuvent admettre cette volonté de casser un tissu social réconfortant fondé sur une mémoire et un vécu spécifique. Le refus d'intégrer la "rue belge" n'est pas politique ou très peu. II relève surtout d'une réalité psychologique : ils ne se sentent pas à l'aise, pas "chez eux" dans les structures "belges". Ces jeunes attendent énormément de cette sociabilité qu'ils ont développée depuis la fin de

\footnotetext{
47 « Note interne (27.9.1950) », archives privées Hertz et Y vonne J ospa, boîte 14, s.d., et A. L apiower, L i ibres enfants du ghetto, 1989, p. 61.

48 | bid., p. 61.

49 «N ote ronéotypée de la Sûreté de I'État concernant I'USJ J ", archives privées Hertz et Y vonne J ospa, boîte 14, s.d.
} 
la guerre. Ils ne peuvent imaginer, parce qu'un lien "tribal" les unit, vivre en dehors de ce milieu qu'ils ont construit. II est à noter que ces relations n'apparaissent en rien aux yeux de plusieurs de ces jeunes de I'USJ J comme une volonté consciente d'affirmation juive bien que, de facto, cette sociabilité soit par essence juive.

Cette décision de dissolution d'une organisation juive communiste est l'aboutissement de cette incompréhension caractéristique des rapports judéo-communistes depuis l'entredeux-guerres. Enfermée dans la crainte qu'ils n'échappent à son contrôle et dans une attitude autoritaire et dogmatique qui fait fi de la réalité "ethnique" et socioculturelle de la "rue juive", la direction du PCB crée les conditions de la rupture.

La Sol assiste donc, sans pouvoir réagir, à la dissolution d'un mouvement de jeunesse dans lequel elle voyait sa relève. C'est dans ce contexte difficile de déception, reniement et désaffection, que la Sol, soumise à de fortes pressions d'intégration à la "rue belge", tentera de se maintenir. P our ce faire, elle maintient les services socio-culturels et place ses colonies de vacances pour enfants au centre de son combat. Dans cette atmosphère politico-idéologique lourde de conséquences, on assiste au recentrage des activités de la Sol sur les sections jeunes, non dissoutes, de l'U SJ J et sur ses centres de vacances.

$A u$ regard des tensions qui caractérisent les relations entre le $P C B$ et ses militants juifs, on est amené à s'interroger sur les éléments qui ont permis de maintenir cette symbiose bien après la L ibération.

Comme nous l'avons déjà indiqué, l'idée d'une Union soviétique émancipatrice des J uifs et libératrice des camps, ainsi que l'image d'un PCB facteur actif et déterminant dans la résistance à l'occupant nazi confèrent à ce parti et à l'idéologie qu'il véhicule un certain crédit. En dehors des milieux juifs les plus politisés, où la confrontation est directe, les tensions liées à la Guerre froide ne sont pas reçues avec la même intensité. II est dès lors possible qu'en milieu juif, en raison d'une empathie aux raisons multiples, le rejet en bloc soit plus progressif et plus tardif.

Il convient également de tenir compte des raisons qui motivent leur adhésion et expliquent leur aveuglement. E lles sont liées à ce qui les a conduits au communisme. Ces militants ont généralement adhéré au communisme dans les années 20 et 30 ou pendant la guerre en raison de 
leur situation socio-économique et de la persécution raciale. Le communisme apparaît donc pour les uns comme une idéologie porteuse d'un projet émancipateur, pour les autres comme le moteur de la R ésistance.

À partir de 1945, le Parti communiste demande à ses militants juifs de s'intégrer. II s'agit donc pour eux de renoncer à un référent identitaire fort et à l'origine de leur adhésion. Le fait qu'ils soient des immigrés de plus ou moins fraîche date est loin d'être un élément anodin. En effet, prendre en considération le niveau d'intégration de ces individus à la société belge et au PCB permet de mieux saisir la pertinence psychologique du lien à la judéité. $P$ arce qu'ils se perçoivent comme des immigrés, ils maintiennent un lien avec une "rue juive" qu'ils n'ont toujours pas quittée.

Le phénomène inverse existe également pour ceux dont le niveau d'intégration à la société belge est élevé ou pour ceux qui ont acquis la nationalité de longue date. R enoncer à la "rue juive" ne pose que peu de problème. N e voit-on pas un R oger $V$ an Praag s'étonner et regretter qu'on distingue encore, en 1957, sur un carton d'invitation de la Sol les orateurs juifs et belges ?50

Depuis la Libération, la politique belge est marquée par la volonté de désavouer les agissements du PCB et de dénoncer les prétentions hégémoniques de Moscou. Cet anticommunisme culmine avec la Guerre froide.

Cette situation pousse les militants communistes à radicaliser leurs discours dans la "rue juive", ce qui entraîne leur isolement. Toutefois, cet isolement n'atteint, dans un premier temps, ni son audience ni sa vitalité. Probablement parce qu'avant l'automne 1948, ce positionnement ne se fait principalement sentir que dans les instances décisionnelles.

Mais la dynamique particulière qui permet à Solidarité juive à la fois d'assumer un engagement idéologique tout en restant ouverte pour l'ensemble de la communauté juive est progressivement minée par la politique du PCB. Tant I'augmentation du contrôle qu'exerce le Parti sur ses militants juifs que l'étouffement et la négation de la spécificité juive viennent mettre un terme à cette difficile symbiose. Ce qui

50 «Courrier adressé par R oger Van Praag à Maggy V olman », 1.10.1957, Solidarité juive, farde 1957, UPJ B. 
apparaissait jusqu'alors comme un « lien informel » se transforme, sous la pression des événements, en dérive autoritaire51. Le conflit larvé avec les sionistes tout comme le bras de fer avec la direction du PCB ne sont perçus que par les plus politisés. Avec le temps, les J uifs communistes soumis à la volonté du PCB de les assimiler, ne bénéficient plus de cette "voie étroite" que leur offraient les revirements stratégiques du PCB (et de Moscou) depuis les années 20. Cette ambiguïté avait permis de concilier ce qui devient, dans la première moitié des années 50 , inconciliable, à savoir l'adhésion au Parti et le maintien d'un rapport à la judéité. A vec le durcissement de la Guerre froide et la cristallisation des opinions, la menace est telle pour un PCB en pleine déliquescence qu'il ne peut plus accepter les "velléités identitaires" de ses militants juifs perçues comme une menace. $L$ 'heure n'est plus à la modération ou au pragmatisme, mais bien au sectarisme.

P oussés dans leurs derniers retranchements, les J uifs communistes, au travers de la Sol, sont doublement victimes. Victimes d'un PCB qui veut mettre un terme à leurs revendications identitaires, mais également victimes d'une communauté juive organisée qui les met au ban. Cet ostracisme communautaire, qui avait germé dès l'état d'urgence passé, est particulièrement perceptible lors des commémorations du soulèvement du ghetto de Varsovie, mais aussi dans la gestion des colonies de vacances de la Sol. Ainsi, Iorsque J ean-Paul H irsch, président de l'asbl "Villa J ohanna", résidence où se tiennent depuis l'été 1946 les colonies de vacances de la Solidarité juive, met un terme au contrat qui le liait à la Sol, il motive sa décision par l'activisme politique et l'endoctrinement idéologique constatables à la Villa J ohanna et qui s'avèrent en contravention avec le contrat de bail 52 .

II est certain que des activités politiques avaient lieu lors des séjours en colonie. Le centre de Middelkerke était devenu un lieu de recrutement pour I'USJJ, dont certains membres, responsables du succès des colonies, étaient des membres du PCB ${ }^{53}$. Le mouvement de jeunesse de la Sol était pour les enfants un prolongement de cette

\footnotetext{
${ }^{51}$ A. Lapiower, L ibres enfants du ghetto, 1989, p. 50.

52 «12.1.1954. Villa J ohanna. Solidarité juive», archives de la Maison H irsch \& Cie-Bruxelles, inventaire 288, liasse 276, AGR . « 18.9.1953. Villa J ohanna. Solidarité juive », ibid.

53 «R apport du service social et colonies pour l'année 1955 », archives Solidarité juive, farde 1955, UPJ B.
} 
atmosphère qu'ils avaient connue durant leur séjour à la colonie de vacances. Dans cette rupture, l'étonnement ne vient pas tant de la réaction elle-même que du temps pris par J ean-Paul $H$ irsch et son conseil d'administration pour réagir. II est peu probable qu'ils n'aient été mis au courant qu'à la fin de l'année 1953 des agissements politiques dont l'établissement était le théâtre. Le directeur de la colonie n'avait-il déjà pas dû faire face à ce type d'accusations ?54 En outre, un document de la Sûreté de l'État, probablement produit au début des années 1950, dénonce cet activisme idéologique55. Comment se fait-il alors que les rapports ne se soient dégradés aussi rapidement qu'à l'automne 1953 ? Alors que la Solidarité juive y voit l'action de milieux « réactionnaires et cléricaux juifs » qui ont tenté de casser la popularité grandissante des colonies de Solidarité juive, il est probable que l'empathie dont bénéficiaient les J uifs communistes n'est, alors que la Guerre froide bat son plein, plus de mises6.

Les tensions politiques liées à la dégradation des rapports EstOuest, la négation du "fait juif" par le Parti et l'absence de réponses valables aux questionnements qui taraudent les J uifs communistes viennent donc mettre un terme à cette entente qui n'a cessé de s'émietter, malgré les sursis stratégiques, depuis la Libération.

Le milieu des années 50 est marqué par la fin des certitudes. La rupture est véritablement consommée et les désaffections se multiplient. Une majorité amère quitte le $P C B$; pour les autres, cette fidélité demeure synonyme de malaise et d'espoirs déçus et se traduit, à terme, par un éloignement. Mais pour certains, elle débouche sur une radicalité politique et une immersion complète en "milieu belge". Se pose alors la question de savoir si le PCB fonctionne comme une "pompe aspirante" qui, tout en vidant la "rue juive" de "ses" militants communistes, favorise par le biais du Parti leur intégration à la société belge. Une étude prosopographique qui analyse à la fois les parcours individuels et la trajectoire collective de ces militants devrait permettre d'y répondre.

\footnotetext{
54 Lettre de J. Istinne, directeur de la Villa J ohanna à E. Haimann (26.7.1950), archives Solidarité juive, farde 1951, UPJ B.

$55 \mathrm{~N}$ ote ronéotypée de la Sûreté de l'É tat concernant I'USJ J , fonds J ospa, boîte 14, s.d. (autour de 1950), CIERL-ULB.

56 Dix ans au service de l'enfance (1945-1955), brochure éditée par Solidarité juive, archives Solidarité juive, farde 1955, UPJ B.
} 
Un rapport de la Sûreté de l'É tat (étonnamment retrouvé dans les archives de Solidarité juive) traduit très clairement l'atmosphère dogmatique qui règne en ce début des années 50 en milieu judéocommuniste. Le rédacteur de ce document relate les propos qu'aurait tenus H ertz J ospa au cours d'une réunion ${ }^{57}$. Conscient des nombreuses désaffections dues à des dérives antisémites en URSS, il réaffirme l'allégeance des camarades au Parti et à I'Union soviétique. Pour H ertz J ospa, le rapport K hrouchtchev en 1956 assume les erreurs de la période stalinienne. En tant que cadre du Parti, il doit faire face à une situation déplorable et est chargé de remotiver un auditoire déboussolé. L'ensemble des documents de la Sûreté de l'É tat que nous avons consultés et qui traitent de l'après rapport $K$ hrouchtchev font écho de "l'inquiétude et du désarroi liés aux purges à caractère antisémite » et à l'attitude proarabe de l'U R SS 58 .

\section{« Le sens de notre existence... »}

Dès septembre 1944, les militants communistes juifs par le biais de la Solidarité juive mettent en place des infrastructures qui doivent permettre à terme un retour à la normale. Leur engagement les fait pleinement participer aux réalisations et aux aspirations de la collectivité juive. En raison de leur activisme dans le processus de reconstruction et de réinsertion socio-économique des J uifs, ils marquent de manière indélébile la collectivité juive bruxelloise durant une brève période après la guerre.

Sa réussite en la matière alimente l'ampleur de son succès et de son audience au sortir de l'Occupation. Aux dividendes du travail social s'ajoute la sympathie accumulée durant les années de guerre en raison de la participation des communistes à la R ésistance. L'évolution de la présence communiste dans la "rue juive" est intimement liée à ses relations avec le PCB et, de ce fait, à l'attitude de la société belge face au communisme et à I'Union soviétique. Dès lors, le contexte anticommuniste belge, le durcissement de la politique de Moscou, les directives du PCB et la gestion catastrophique de la "question juive" par ce dernier sont autant de facteurs explicatifs de leur progressive

\footnotetext{
57 «J uifs, Solidarité juive », Sûreté de l'É tat (63), archives de Solidarité juive, farde 1956, UPJ B. $58 \mid$ bid.
} 
marginalisation dès la fin des années 1940 au sein de la "rue juive". Devoir constamment louvoyer afin d'assumer les revirements politiques liés aux "stratégies" du PCB et à son alignement sur les directives de Moscou fait peser sur la Sol un certain discrédit et éloigne définitivement la majorité de ceux qui, sans pour autant y adhérer, avaient manifesté une sympathie certaine pour le PCB. L'amertume des jeunes de I'USJ J suite à la guerre d'I ndépendance israélienne, la désillusion de ceux qui rentrent de Pologne, des échos d'antisémitisme en Union soviétique et dans les républiques populaires, le rapport $K$ hrouchtchev quelques années plus tard, ainsi que les événements polonais et hongrois, sont autant d'éléments qui entament l'idéal de nombreux communistes juifs. Le contexte politique et une "question juive" systématiquement réduite par le PCB à de l'activisme sioniste et des conflits d'intérêts précipitent la fin d'une présence significative des communistes dans la "rue juive" à partir du milieu des années 50.

De cette tension émerge l'ambiguïté du rapport que ces militants entretiennent avec l'utopie communiste. Les déchirements nés de l'opposition entre leur immersion dans la modernité issue de leurs choix idéologiques et leur ancrage dans la "rue juive" à laquelle ils ne peuvent ni ne souhaitent se soustraire, s'ils étaient déjà présents au cours de l'entre-deux-guerres, persistent au sortir de l'Occupation ${ }^{59}$. I Is confirment par là que l'héritage culturel et historique est un facteur de différenciation qui n'est pas uniquement formel. Ce schéma sera toutefois remis en cause suite au processus de reconstruction qui favorisera, à terme, une mobilité socio-économique et géographique. Ces J uifs communistes, à la judéité affirmée, ont été plus juifs qu'ils ne le voulaient ou ne le pensaient. Cette situation, source de tensions, s'est prolongée après la guerre et malgré le PCB. II en va de même pour leur adhésion au communisme. Ils continueront, bien après la faillite de celui-ci, à soutenir la politique du PCB et le régime de Moscou parce qu'ils avaient, sans doute bien plus que les militants "belges", investi cette idéol ogie d'attente liée à leur condition de J uifs ${ }^{60}$.

Au-delà du travail de recréation du tissu social s'affirme de manière plutôt paradoxale en milieu communiste une permanence juive,

\footnotetext{
59 R. Van D oorslaer, « Une identité communiste judéo-bessarabienne. L es J uifs de B elgique dans la guerre civile espagnole», dans J .-P. Schreiber (éd.), H ertz J ospa..., 1997, pp. 47-50.

60 | bid. , 1997, p. 50.
} 
expression d'une identité forte et assumée. La détérioration des relations entre le $P$ arti et les J uifs communistes est particulièrement parlante. Elle s'opère sur fond d'affirmations identitaires et rend perceptible la difficile - voire impossible - conciliation entre l'idéal politique qui se traduit par une allégeance indéfectible au Parti et au régime soviétique et l'attachement à la judéité. L'affirmation d'une spécificité juive, évidence objective dont le PCB n'a que faire, apparaît non comme l'expression d'un repli, mais bien comme la volonté de préserver et de transmettre une expérience historique et culturelle singulière. Comme le résume si explicitement un rédacteur du journal Unzer K amf : "Attachés à notre identité non par entêtement ni par habitude, [mais parce qu'elle donne] le sens de notre existence et peutêtre la force de supporter et de surmonter toutes les difficultés, notre culture est le pont qui, au travers du présent, relie notre passé au futur. ${ }^{61}$ Mais dès lors, se pose en milieu juif progressiste la difficile question du contenu de cette identité. II est probable que pour la génération d'avant-guerre, majoritairement issue du Y iddishland, cette spécificité ne se pose pas, elle s'impose. Mais pour les générations suivantes, une série de repères sont indispensables pour tenter de saisir les aspects de cette judéité. I ntervient alors un ensemble de paradigmes. L'enfance juive est investie de toutes les espérances de l'aprèsguerre. En raison d'un légitime désir de "normalisation", ils sont nombreux à s'engager dans le communisme et dans la cause soviétique jusqu'à nier l'évidence. Enfin, la mémoire de la Résistance et de la lutte contre le fascisme répond à certaines carences identitaires et offre une légitimité à cette spécificité judéo-communiste. En effet, en raison de l'attitude du $P C B$, cette mémoire va progressivement devenir la seule valeur liée au vécu juif récent qui puisse être assumée et transmise sans trop de contradictions.

Au lendemain de la Libération, l'identité des J uifs communistes demeure ambiguë. Seuls des procédés complexes permettent d'assumer des contradictions qui, en raison de la conjoncture, ne cessent de se multiplier. Cette situation est propice à l'isolement des J uifs communistes tant au sein des structures du PCB que dans la "rue juive". La dialectique entre aspirations universalistes et identité juive

61 « Comment peut-on et doit-on résoudre la question juive ? », dans U nzer K amf, n² 20, 6.1.1945, p. 2 (traduit du yiddish par J oseph Szyster). 
engendre des tensions auxquelles ni le Parti, enfermé dans une attitude dogmatique, ni la communauté juive, en reconstruction et soumise à de fortes pressions, ne peuvent répondre.

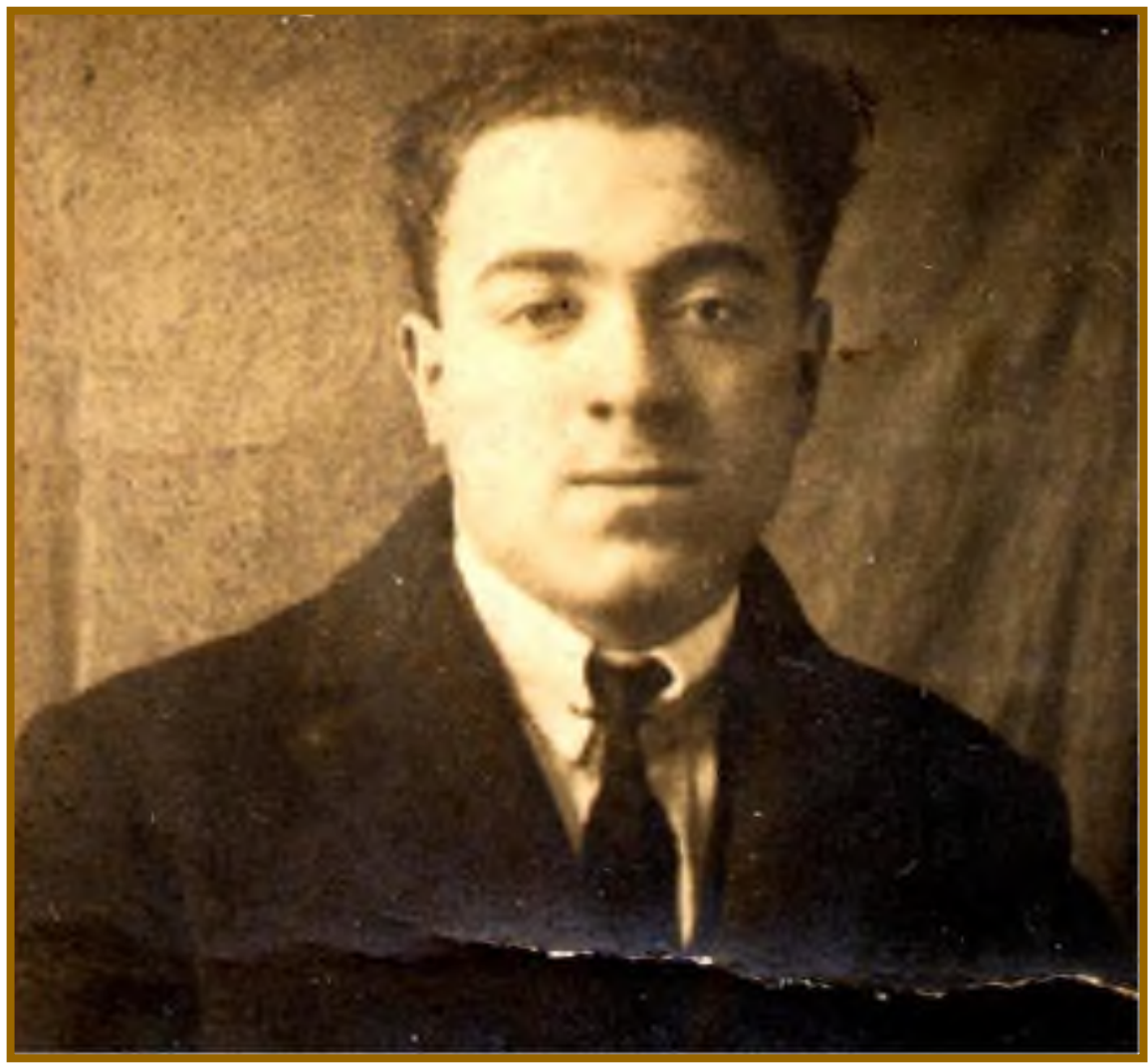

H ertz J ospa.

(c) SPF - OÉ 


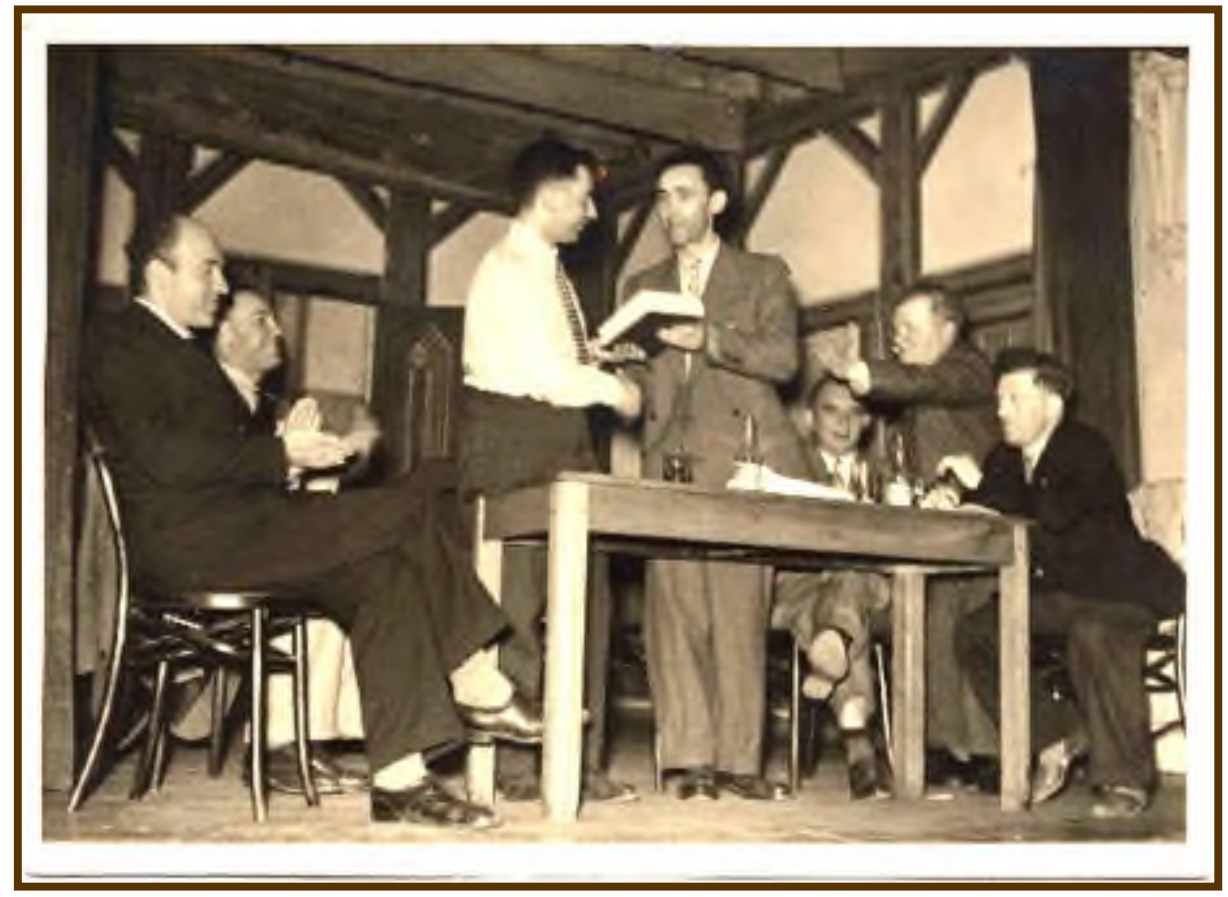

A ssis, à gauche: $\mathrm{H}$ ertz J ospa et B er Szyster.

Debout, à droite : R ichard W olman. Assis, à droite : J acob Gutfrajnd.

(c) UPJ B 